\title{
Evolución de la política de cambio climático en Colombia*
}

\author{
Evolution of Climate Change Policy in Colombia
}

\author{
Luis Magin Guardela Contreras a \\ Universidad del Norte, Colombia \\ lguardela@uninorte.edu.co \\ ORCID: https://orcid.org/0000-0002-6902-417X
}

DOI: https://doi.org/10.11144/Javeriana.vj69.epcc

Recibido: 06 Febrero 2020

Aceptado: 01 Junio 2020

Publicado: 21 Diciembre 2020

\section{Resumen:}

El presente artículo analiza la política pública sobre cambio climático, al acotar los avances en la consolidación de una visión y, en consecuencia, la formulación de la misma. Para lograr este objetivo se utilizó una metodología de corte jurídico, analítico y descriptivo con enfoque predominantemente cualitativo, cuyo insumo primario fueron las medidas jurídico-administrativas adoptadas por los estamentos políticos-administrativos en relación con el objeto de estudio. Se concluye que para hacer efectiva la visión preventiva (mitigación) y adaptativa para afrontar el fenómeno, se debe realizar: a) una articulación efectiva entre las entidades que integran la gestión pública del Estado colombiano; b) la inclusión efectiva de rubros presupuestales sobre cambio climático en las entidades públicas de todo los niveles, en razón de la transversalidad de la política pública, y; c) el reforzamiento de las apuestas en las variables de mitigación y adaptación en los instrumentos de planificación del territorio, desarrollo socioeconómico y la gestión del riesgo de desastres.

Palabras clave: Cambio Climático, Mitigación, Adaptación, Política Pública, gestión del riesgo de desastres.

\section{Abstract:}

This article analyzes the public climate change public, keeping within bounds the advances in the consolidation of a vision and, in consequence, its formulation. A legal, analytical, and descriptive methodology with a predominantly qualitative approach was used, whose main contribution was the administrative-legal measures adopted by the political categories concerning the object of study. Therefore, it should be emphasized that to achieve effective visions of the mitigation and adaptation to the phenomena of climate change implies: a) An effective articulation of the entities that integrate the public management of the Colombian State; b) The effective inclusion of budget items related to climate change in the public entities at all levels, by reasons of the transversality of public policy; c) The reinforcement on the bets in the mitigation and adaptation variables in the instruments of territorial planning, socio-economic development, and disaster risk management.

Keywords: Climate Change, Mitigation, Adaptation, Public Policy, Disaster Risk Management.

\section{Introducción}

El mundo tardó dieciocho siglos para sumar mil millones de habitantes, hoy, cada doce años alcanza ese número ${ }^{1}$. En ese sentido, podríamos decir que la Revolución Industrial aportó incontables sucesos a la humanidad, tanto positivos como negativos, entre estos últimos, la explosión demográfica del siglo XVIII, que aumentó las exigencias y demandas de recursos naturales para la satisfacción de necesidades ${ }^{2}$.

Si bien la teoría de la transición demográfica ${ }^{3}$ revela que en la actualidad el planeta tiene las tasas de natalidad más bajas de su historia, el crecimiento natural de la población ha llevado a rebasar su capacidad de carga ${ }^{4}$ o sobrepasarla ${ }^{5}$, tal como fue advertido desde mediados del siglo $\mathrm{XX}^{6}$.

A través de Earlich ${ }^{7}$ y sus álgidas polémicas con Julian L. Simon se sabe que la sobrepoblación produce efectos que perturban la capacidad de resiliencia del planeta: recursos que se explotan en demasía hasta agotarlos; aumento en la demanda y, por tanto, consumo ineficiente; contaminación en todas sus formas;

Notas de autor

\footnotetext{
a Autor de correspondencia. Correo electrónico: lguardela@uninorte.edu.co
} 
conflictos ambientales y socioambientales por acceso y uso y; por supuesto, fenómenos globales como el agotamiento de la capa de ozono y el cambio climático.

En todo esto, las ciudades juegan un papel crítico. Así como el siglo XVIII fue considerado como el de la explosión demográfica, el Siglo XX fue el de la urbanización ${ }^{8}$; vivimos hoy en un entorno planetario predominantemente urbanizado.

Desde agosto de 2007, más del 50\% de la población mundial vive en ciudades ${ }^{9}$, las más pobladas, lastimosamente, localizadas en los países con mayor vulnerabilidad. Sin embargo, y de manera paradójica, allí donde muchas cosas faltan o no se producen de manera principal, es donde se generan los impactos que provocan el cambio climático.

Para el caso local, el país tiene una población total de cuarenta y ocho millones doscientos cincuenta y ocho mil cuatrocientos noventa y cuatro $(48.258 .494)^{10}$ habitantes y, al igual que el planeta y el continente en el cual se ubica, también está urbanizada: cerca del $77 \%$ de la población ${ }^{11}$ habita hoy en una ciudad.

De acuerdo con el Consejo Nacional de Política Económica y Social (en adelante CONPES) ${ }^{12}$, mientras que en 1951 la población urbana del Colombia equivalía al 39\% de la población total, la economía era predominantemente agrícola, solo había seis ciudades que superaban el umbral de los cien mil habitantes y carecía de urbes que llegaran al millar de estos. Las proyecciones muestran que para 2050 los centros urbanos del país alcanzarán los 52,6 millones de habitantes, lo que representa el 88,3\% del total proyectado por la CEPAL (2019) ${ }^{13}$ además, tendrá setenta y nueve ciudades con más de 100.000 habitantes y siete con más de un millón ${ }^{14,15}$.

También, al igual que ocurre con la distribución demográfica mundial, en Colombia las principales actividades aportantes de gases de efecto invernadero (GEI) se dan como consecuencia de la actividad antrópica urbana: movilidad, hogares, industria, etc. ${ }^{16}$. Pese a que Colombia continúa sin hacer aportes significativos a la bolsa mundial de $\mathrm{GEI}^{17}$, sí tendrá que efectuar esfuerzos importantes de mitigación, y aún más en su adaptación a los efectos del cambio climático, como todos los países parte del Acuerdo de Paris.

De acuerdo con el Banco de Desarrollo de América Latina ${ }^{18}$, Colombia tienen un índice de vulnerabilidad climática medio (puesto 20 entre 33), comparte esta categoría con Venezuela y Ecuador, junto a Bolivia y Belice. La razón por la cual Colombia no está en el top de dicha lista se debe a que, en teoría, está mejor preparado para enfrentar el fenómeno al haber configurado un sistema nacional de mitigación y adaptación.

Con todo, el panorama actual justifica realizar un análisis de la evolución de la visión que Colombia ha tenido frente al cambio climático, mostrar los avances y también retrocesos en la configuración de una visión y, en consecuencia, de una política pública para hacerle frente.

Se acudió a una metodología de corte jurídico, analítico y descriptivo con enfoque cualitativo, cuyo insumo fueron las medidas jurídico-administrativas adoptadas por Estado en relación con el cambio climático, utilizando como técnica el análisis jurídico de las fuentes del derecho, especialmente las leyes y actos administrativos expedidos sobre la materia y el análisis documental de la información oficial.

\section{Evolución en la concepción del cambio climático en el mundo y Colombia}

\section{Contexto internacional}

Inicialmente, es necesario analizar la evolución de los compromisos internacionales sobre la temática para, posteriormente, explicar el contexto y los principales hechos climáticos que han marcado la transformación en la concepción y visión sobre el tema.

La Primera Conferencia Mundial sobre el clima tuvo lugar durante 1979 en Ginebra, Suiza, citada por la Organización Meteorológica Mundial (en adelante OMM), cuyo resultado fue una exhortación a los Estados 
alrededor del mundo a vigilar y monitorear las acciones antropocéntricas que pudieran causar alteraciones virtuales en el clima al adoptar el Convenio sobre Contaminación Atmosférica Transfronteriza a Gran Distancia ${ }^{19}$.

En esa misma conferencia se estableció el Programa Mundial sobre el Clima, como resultado de los esfuerzos del Consejo Internacional para la Ciencia, la Organización Meteorológica Mundial y la Comisión Oceanográfica Intergubernamental de la Unesco ${ }^{20}$. El objetivo de este programa es generar información científica para ayudar en la comprensión del sistema climático, sus procesos y, por supuesto, sus cambios. El programa tiene amplias líneas de campos de acción que complementan los trabajos del Grupo Intergubernamental de Expertos sobre el Cambio Climático (IPPC, por sus siglas en inglés) en sectores como estudio de la atmósfera, los océanos, glaciares, y la superficie de la tierra ${ }^{21}$.

Precisamente, en el año de 1988 el Programa de Naciones Unidas para el Medio Ambiente creó el referido IPPC como un organismo técnico-científico encargado de evaluar la ciencia del cambio climático, sus impactos, riesgos, y las formas de mitigación y adaptación, y a partir de ello crear fundamentos para la formulación de las políticas estatales relacionadas con el clima ${ }^{22}$.

A la fecha, el IPCC ha realizado cinco informes de evaluación emitidos en 1990, 1995, 2001, 2007 y 2014 , y se han reunido en 48 oportunidades. Recientemente, tuvo lugar la última en Kioto (Japón) durante mayo del 2019, donde presentaron la actualización de la metodología para el levantamiento de inventarios de GEI como principal novedad ${ }^{23}$. Por su parte, se espera que el sexto informe del IPCC esté listo para 2021, cuando se haya podido hacer un balance de la situación mundial y, sobre todo, del cumplimiento de las medidas impulsadas en el Acuerdo de Paris que comenzará a regir a partir de $2020^{24}$.

Estos informes presentados han sido los primeros en confirmar con evidencia científica las consecuencias del cambio climático, lo que llevó a la comunidad internacional a negociaciones para la elaboración de una convención sobre el clima, que ocurrió en mayo de 1992 en Nueva York y derivó en la adopción de la Convención Marco de las Naciones Unidas sobre el Cambio Climático (en adelante CMNUCC) ${ }^{25}$. Los documentos resultantes fueron suscritos por 154 países en la cumbre de la ONU para el Medio Ambiente y el Desarrollo, mejor conocida como Cumbre de Río de Janeiro, en junio de ese mismo año.

La CMNUCC tuvo como objetivo principal la creación de medidas e instrumentos jurídicos efectivos para viabilizar la estabilización de las concentraciones de GEI en la atmósfera a un nivel que impidiese interferencias antropógenas peligrosas para el sistema climático. En el numeral 4 de su artículo 7, consagró el régimen de sesiones ordinarias y extraordinarias de las partes ${ }^{26}$ (COP, por sus siglas en inglés), con el fin de revisar su aplicación y tomar decisiones ágiles, señalando que las sesiones ordinarias se celebrarían cada año, y las extraordinarias cada vez que la Conferencia lo considerase necesario.

Teniendo en cuenta las medidas y decisiones adoptadas hasta hoy, de las 24 conferencias realizadas, las más importantes son dos. La primera es la COP 3 (Kioto, Japón, 1997), en la que se otorgaron dientes a los mecanismos de la CMNUCC a partir de dos tipos de medidas: las obligatorias, que consisten en el establecimiento de metas de reducción de emisiones para los países con mayor generación de dióxido de carbono $\left(\mathrm{CO}_{2}\right.$, considerado el GEI equivalente), el cual debía ser reducido en un 5\% tomando como referencia las producidas en 1990 y debían producirse en el período entre 2008 y $2012^{27}$.

El otro tipo de medidas fueron las denominadas de flexibilidad o de mercado: un conjunto de normas y mecanismos para promover el comercio de emisiones atmosféricas entre Estados industrializados con metas de reducción de emisiones, así como la cooperación entre Estados con metas de reducción y países en vías de desarrollo sin metas definidas. Tres fueron los mecanismos ideados para cumplir con el propósito: el mercado del carbono, el intercambio de certificados de reducción o CERTS y el mecanismo de desarrollo limpio $(\mathrm{MDL})^{28}$. 
Sin duda, el Protocolo de Kioto fue la base para la promulgación de leyes y políticas de los Estados, orientó la toma de decisiones y medidas para el cumplimiento de los compromisos adquiridos y la reducción de los GEI.

La segunda conferencia de importancia es la COP 21 (París, Francia, 2015), bautizada como la conferencia poskioto: en esta se buscaba establecer nuevos lineamientos para luchar contra el cambio climático, al haber expirado el tiempo pactado (2008-2012) para reducir las concentraciones de GEI en el mundo ${ }^{29}$.

Los compromisos de Kioto no se cumplieron y desde la COP 15 (Copenhague, Dinamarca, 2009) se sabía que no se alcanzarían; los países con metas definidas de reducción habían sobrepasado sus topes. Los años posteriores a la COP 15 fueron difíciles con respecto a la negociación de un acuerdo después de 2012, teniendo en cuenta el fracaso en el acatamiento de los acuerdos anteriores.

El artículo 2 de este acuerdo señala sus objetivos claramente, los cuales se circunscriben a la erradicación de la pobreza y la consolidación del desarrollo sostenible mediante la mejora en las capacidades de adaptación y el equilibrio financiero global, para así garantizar el aumento de temperatura por debajo de los $2^{\circ} \mathrm{C}$ frente a los niveles preindustriales ${ }^{30}$.

Por otra parte, no puede dejar de mencionarse que en la COP 22 se encuentran las formulas, métodos y recursos para ir abonando el camino hacia las metas trazadas después de examinada la situación de los países en vía de desarrollo para el cumplimiento de los acuerdos y se instaló el sistema mundial de observación del $\operatorname{clima}^{31}$.

Posteriormente, en la COP $23^{32}$ se evaluaron los procesos técnicos promovidos en materia de adaptación y mitigación al cambio climático, a la par que se escudriñó sobre el funcionamiento del comité delegado para el perfeccionamiento de la próxima entrada en vigor del Acuerdo de París.

En La COP 24 de Katowice, Polonia, se discutió la necesidad de reforzar el desarrollo y transferencia de tecnología para la gestión climática y se establecen medidas para colocar al día a las naciones en mora de cumplir con los presupuestos de desarrollo para la venidera regencia del Acuerdo de París ${ }^{33}$.

A pocos días de llevarse a cabo la COP 25 en la ciudad de Santiago de Chile, el 2 de diciembre de 2019, el presidente de dicha nación decidió no ser país anfitrión del evento debido al estallido de una crisis social, económica y política. Por lo tanto, se eligió a Madrid como su reemplazo.

\section{Contexto Colombiano}

Al ritmo del contexto internacional, Colombia se ha movido y se sigue moviendo en relación con el cambio climático, los períodos de evolución en la visión nacional frente al fenómeno coinciden con los avances logrados en el contexto global. Sin embargo, los avances que se presentan tanto a nivel internacional como nacional son respuesta a los efectos del cambio climático.

En virtud de lo anterior, se señalan tres períodos en relación con los avances de la política de cambio climático en Colombia, hasta su expedición. Un primer período comprende de 1994 a 2010. En esta fase, la visión del país frente al cambio climático fue esencialmente económica y estuvo enfocada a sacar provecho económico de los proyectos que pudieran formularse a partir de los mecanismos implementados en los acuerdos y conferencias internacionales sobre la materia.

Un segundo momento cubre el primer período de Juan Manuel Santos y su Plan de Desarrollo "Prosperidad para Todos" (2010-2014): en este período al presidente le correspondió vivir un severo invierno como consecuencia del fenómeno de "La Niña" que azotó el país a finales del mismo año. A partir de los impactos y los desastres vividos de esta situación, la visión frente al fenómeno comenzó a virar hacia una mayor atención a la adaptación y a la gestión del riesgo, los costos asociados a los riesgos climáticos pueden ser devastadores para la población y la economía nacional ${ }^{34}$. 
El último período (2014-2018) se enmarca en el segundo periodo del gobierno Santos y su Plan de Desarrollo “Todos por un Nuevo País”, que se caracterizó por la necesidad y obligación como país de adherir y cumplir con los nuevos compromisos internacionales como el Convenio de Paris y los objetivos de desarrollo sostenible al empalmar con la Política Nacional de Cambio Climático expedida a finales del año 2017 y la expedición de la Ley 1931 de 2018, por la cual se establecen la directrices para la gestión del cambio climático.

\section{Primerperiodo}

Las bases sobre las cuales se erige actualmente la Política Nacional de Cambio Climático fueron abonadas normativamente a partir de la expedición de la Ley 164 de $1994^{35}$ y por el influjo de la CMNUCC ${ }^{36}$ que, a su vez, corresponde a los cimientos otorgados por la Declaración de Río (1992).

Se dio la ratificación del Protocolo de Kioto $(1997)^{37}$, dándole así continuidad al camino ya trazado, suscitado por la creciente preocupación de la comunidad internacional frente al incremento en las concentraciones de GEI y sus posibles efectos catastróficos sobre el cambio climático.

A pesar de la ratificación de los instrumentos internacionales, la visión que se tenía en ese momento frente al cambio climático era, como se señaló, predominantemente económica, enfocada en la posibilidad de fortalecer la economía del país a partir del uso de los mecanismos de flexibilidad incorporados en el protocolo, en particular, el Mecanismo de Desarrollo Limpio (MDL) ${ }^{38}$.

En tal atmósfera se dio la primera "Comunicación nacional ante la convención marco de las naciones unidas sobre el cambio climático” en 2001, liderada por el IDEAM, el cual informó el inventario nacional de GEI del periodo 1990-1994, efectuado a partir de la metodología dada por el IPCC. Se enfatizó en la emisión de dióxido de carbono (CO.), producto del uso de combustibles fósiles y la derivada por uso de biomasa. Para el primer año analizado se dio una concentración total de 129.368.4 Gg (gigagramos), distribuidos en 63.510.4 $\mathrm{Ggde} \mathrm{CO}_{2}, 41.241 .1 \mathrm{Gg}$ de Metano (CH.) y $24.617 \mathrm{Gg}$ de Óxido Nitroso (N.O), mientras que para la última parte del periodo hubo un incremento de77.103 Gg de CO., $44.450 \mathrm{Gg}$ de CH. y 28.313 Gg N.O, para un incremento global de $149.867 \mathrm{Gg}^{39}$.

En procura al cumplimiento de los deberes suscritos, el gobierno elaboró los "Lineamientos de Política de Cambio Climático" en 2002, bajo la directriz del Ministerio de Medioambiente y el DNP, en el que explicaron nociones como cambio climático global, el efecto invernadero, la vulnerabilidad del país frente a dicho fenómeno. Además, se esbozaron medidas de respuesta ante la ratificación de los instrumentos internacionales antes mencionados, a la vez que comentan las oportunidades que estos representan en cuanto a iniciativas de MDL.

Lo anterior se enmarcó en cuatro objetivos: 1) adaptarse a los impactos del cambio climático, 2) reducir de emisiones por fuentes y absorción sumideros de GEI, 3) atenuar el impacto de los compromisos del protocolo de Kioto en la exportación de combustibles fósiles y 4) promover la investigación sobre el cambio climático ${ }^{40}$.

En el año 2003 el Consejo Nacional de Política Económica y Social expidió el CONPES 3242 den donde se incluye la "Estrategia Institucional para la Venta de Servicios Ambientales de Mitigación del Cambio Climático", como una de las alternativas consagradas en el Protocolo de Kioto ${ }^{41}$ para la consecución de la meta de aminoramiento de emisiones de GEI. En tal instrumento se dispone la consolidación de una oferta de reducciones verificada y el asentamiento de un mercado internacional donde transar dichas operaciones. Dichas operaciones deben estar centradas en MDL, en armonía con los beneficios tributarios para tal segmento económico previstos en la Ley 788 de $2002^{42}$.

Dos años después, se expidieron las Resoluciones 0453 y 0454 de 2004 por parte del ministerio de medioambiente, normativas que adoptaron los principios, condicionamientos y directrices para el establecimiento lineamientos para la aprobación de iniciativas de disminución de emisiones de GEI a través 
de MDL, así como regular la operatividad del Comité Técnico Intersectorial de Mitigación del Cambio Climático que creó el Consejo Nacional Ambiental ${ }^{43}$ para evaluar los proyectos MDL.

Posteriormente, durante el segundo mandato de Álvaro Uribe (2006-2010), en su Plan de Desarrollo "Estado Comunitario: Desarrollo para Todos", se estipuló como prioritaria la labor de facilitación de los proyectos de MDL de manera conjunta con la creación y fortalecimiento de medidas para la adaptación del país al cambio climático ${ }^{44}$.

Durante dicha administración, ocurrió la presentación de la "Segunda comunicación nacional ante la convención marco de las naciones unidas sobre el cambio climático" en 2009, en la que se describen los aspectos geopolíticos del país y se hace una radiografía de la oferta ambiental de los territorios. Como componente central se expuso el inventario nacional de GEI para el periodo 2000-2004. En este último año del periodo se calcularon $180.010 \mathrm{Gg}$ de emisiones, representadas en un $50 \%$ por dióxido de carbono (CO.), 30\% de Metano (CH.) y un 19\% de Óxido nitroso (N.O). El 1\% restante estuvo compuesto por los hidrofluorocarbonos (HFC), perfluorocarbonos (CFC), halocarbonos y hexafluoruro de azufre (SF. $)^{45}$.

De igual manera, presentó un estudio sobre los rasgos fisicobióticos y socioeconómicos del país de cara a la especificación de los niveles de vulnerabilidad ante los efectos del cambio climático fundamentado en las alteraciones hidrometeorológicas asociadas a este ${ }^{46}$.

\section{Segundo Periodo}

Dos años después, con la expedición de la Ley 1450 de 2011 (Plan de Desarrollo 2010-2014) "Prosperidad para Todos”, se implantó, como propósito gubernamental para dicho lapso, incorporar como lineamiento transversal a todas las esferas del quehacer nacional la idea de una sociedad para la cual la sostenibilidad ambiental, la adaptación al cambio climático, el acceso a las nuevas tecnologías y el desarrollo cultural sean principios de equidad para las futuras generaciones ${ }^{47,48}$.

En la misma normatividad se dispuso el acompañamiento del DNP a los entes territoriales mediante el programa para la creación y robustecimiento de capacidades institucionales para el desarrollo territorial, entre cuyas acciones se halla la asistencia técnica en gestión del riesgo por cambio climático ${ }^{49}$. Es importante notar que Ley 1450 de 2011 delega la formulación del Plan Nacional de Adaptación al Cambio Climático (PNACC) al DNP en coordinación con el por entonces Ministerio de Vivienda y Desarrollo Territorial ${ }^{50}$.

En ese mismo año se expidió el CONPES 3700, por el cual se formuló la "Estrategia institucional para la articulación de políticas y acciones en materia de cambio climático en Colombia”, cuyo cometido fue coordinar la inclusión de la variable de cambio climático en las instancias de deliberación técnica y política institucional, a fin de reducir la exposición del país a los efectos de este fenómeno y concurrir en la construcción de una visión de desarrollo económico sostenible ${ }^{51}$.

En el periodo 2012-2013 se dio la expedición del documento técnico "Plan Nacional de Adaptación al Cambio Climático. Adaptación Bases Conceptuales: marco conceptual y lineamientos”. Este instrumento integra una contextualización nacional, cuyo epicentro es la determinación de responsabilidades conforme a los antecedentes y el impacto de los planes de trabajo a adelantar; hace una reseña de la conexidad conceptual entre vulnerabilidad, amenaza climática, gestión del riesgo y adaptación; presenta una fundamentación científica para adelantar acciones de adaptación nacional con respecto a la incidencia de los sectores productivos y la afectación a las poblaciones vulnerables; $y$, finalmente, propone pautas para una planificación adecuada del proceso de adaptación nacional al cambio climático a partir de criterios como costoeficiencia, la implementación multinivel articulada, productividad, protección de focos humanos vulnerables y complementariedad con la gestión del riesgo de desastres ${ }^{52}$. 
A partir de este plan, se formuló el instrumento "Hoja de ruta para la elaboración de los planes de adaptación dentro del Plan Nacional de adaptación al cambio climático", en el cual se plasmaron orientaciones a entidades territoriales y sectoriales para la elaboración de acciones de adaptación al cambio climático, ajustadas a sus condiciones particulares y en correspondencia con otros instrumentos tanto de gestión como de planificación ${ }^{53}$.

Consecuentemente, se sugirió como esquema procedimental: 1) diagnóstico y preparación; 2) administración de insumos para la sostenibilidad en escenarios de cambio climático; 3) la determinación de medidas de adaptación, así como su priorización; 4) la estructuración y posterior implementación de estas (medidas de adaptación); y 5) monitoreo y evaluación de los resultados arrojados ${ }^{54}$.

\section{Tercer Periodo}

Posteriormente, para el año 2015, la variable de cambio climático también fue abordada al interior del Plan Nacional de Desarrollo “Todos por un Nuevo País” al figurar en el plan plurianual de inversiones. Sin embargo, el rubro asignado a esta variable se podría considerar marginal, pues fue de $\$ 5.119 .649$ como indicador del estándar de crecimiento verde a título de la consecución de un crecimiento resiliente y el aminoramiento de la vulnerabilidad frente al riesgo de desastres y el cambio climático ${ }^{55}$.

Allí también se le dio competencia al Fondo de Adaptación para estructurar y ejecutar proyectos de reducción del riesgo y adaptación al cambio climático en el marco del Sistema Nacional de Gestión del Riesgo de Desastres, en aras del robustecimiento de las funciones de dicha instancia y reducir la vulnerabilidad fiscal del Estado ${ }^{56}$.

En esta normatividad se impone a los ministerios de Hacienda, Agricultura y Desarrollo Rural, Minas y Energía, Transporte, Salud y Protección Social, Vivienda, Ciudad y Territorio y Comercio, Industria y Turismo la implementación de los Planes de Acción Sectorial (PAS) de Mitigación para el Cambio Climático, los cuales contaron con metas cuantitativas de reducción de emisiones de GEI a corto (año 2020) y mediano plazo (años 2025 o 2030) $)^{57}$.

Luego, se elaboró la “Tercera Comunicación Nacional de Cambio Climático a la CMNUCC” en el 2015, la cual expone un consolidado del inventario de GEI durante el periodo 1990-2012 mediante estimaciones comparadas con las emisiones mundiales, analiza los factores de vulnerabilidad territorial, compilan las acciones de mitigación y adaptación adelantadas y se sugieren caminos de acción para solventar las carencias de financiación ${ }^{58}$.

En el 2016 se estableció el Sistema Nacional de Cambio Climático (en adelante SISCLIMA), un agregado de instituciones estatales, privadas y entidades sin ánimo de lucro, así como de políticas, programas, planes, reglamentaciones e instrumentos asociados a cambio climático cuya aplicación obedece a la gestión en las labores de la mitigación de GEI y la adaptación a las implicaciones territoriales del cambio climático en el país ${ }^{59}$.

El objetivo de esta estructura político-administrativo-intersectorial en materia medioambiental, pero subespecializado en la variable de cambio climático, es la articulación y evaluación de las acciones para la adaptación al cambio climático y la mitigación en la emisión de GEI. Toman como referencia la corresponsabilidad en la asunción de la administración de dicha gestión entre las entidades del orden nacional, departamental y local con el sector productivo e, igualmente, la incidencia de la sociedad civil ${ }^{60}$.

Sobre este sistema, es preciso señalar que su formulación data de cinco años atrás a su normativización, puesto que es reseñado en el marco de la estrategia institucional que se sugiere en el CONPES 3700, propuesto como consecuencia del diagnóstico adelantado sobre los retos y oportunidades generados por el cambio climático en el país; la necesidad de transferir responsabilidades directas en cabeza de los actores económicos 
del mercado, sociedad civil; y comprometer a los entes territoriales en la realización de la mitigación y adaptación al cambio climático alrededor del eje de la complementariedad ${ }^{61}$.

Sin embargo, pese a disponer de un término razonable de seis meses para su materialización vía acto administrativo y posterior entrada en vigor ${ }^{62}$, el SISCLIMA no se produjo sino hasta casi cinco años después. El primero fue aprobado el 14 de julio de 2011 y el segundo fue expedido el 24 de febrero de 2016.

En la especificación del mandato del SISCLIMA sobresalen los planes y estrategias de cambio climático articulados de forma holística con procesos económicos, sociales y ambientales. El SISCLIMA parte de las condiciones prioritarias para la consecución de un desarrollo sustentable, la erradicación de la pobreza y la sostenibilidad de los recursos naturales. Así mismo, se basa en la participación ciudadana en un marco democrático para la toma de decisiones relacionadas con la gestión del cambio climático y las funciones, tanto de monitoreo como de evaluación de los deberes en materia de adaptación al cambio climático y mitigación de GEI ${ }^{63}$. En este segmento, también destaca como medular la ratificación del Acuerdo de París por parte del Congreso de la República a través de la Ley 1844 de 2017.

\section{Política Pública de Cambio Climático}

Todo este recorrido de esfuerzos gubernamentales, modernización institucional, expedición legislativa y emprendimiento de acciones para afrontar las labores de mitigación y adaptación al cambio climático condujeron a la elaboración de la Política Nacional de Cambio Climático en 2017, como condensadora de los objetivos de sinergia entre los sectores estatales de diverso orden público en general e integrantes de la agenda productiva nacional. Lo anterior, con el fin de encarar el desarrollo territorial con una perspectiva de mitigación y adaptación al cambio climático, y abordar el diseño de medidas en la materia, la relevancia de la articulación direccionada a un crecimiento sustentable, los parámetros de competitividad de la nueva economía del clima, y la gestión de los aspectos sensibles en la intersección de la gobernanza ambiental y la dinámica mercantil ${ }^{64}$.

Posteriormente, se expidió la Ley 1931 de $2018^{65}$, estatuto que busca posicionarse como el referente integrador de la fragmentada legislación ambiental en la variable subexamine para la toma de decisiones asociadas a la administración de cambio climático de personas públicas y privadas, al fomentar la concurrencia de las instancias nacionales, departamentales, locales y de autoridades ambientales para la mitigación de GEI. Igualmente, busca la reducción de la vulnerabilidad de la población y de los hábitats del país ante manifestaciones de este fenómeno en procura del establecimiento gradual y mancomunado — conforme a los principios de coordinación, corresponsabilidad e integración dispuestos en sus enunciados rectores- de una economía competitiva, sustentable y baja en carbono ${ }^{66}$.

Así mismo, la ley normativiza los denominados Planes Integrales de Gestión del Cambio Climático Territoriales (PIGCCT) al concebirlos como instrumentos de identificación, evaluación, priorización y toma de medidas relacionadas con el cambio climático por parte de los entes territoriales. Todo esto, enmarcado en la meta global de reducción de $\mathrm{GEI}^{67}$.

\section{Objetivos y Responsables}

El cometido que persigue la Política Nacional de Cambio Climático es incorporar la gestión del cambio climático en la toma de decisiones tanto en el ámbito oficial como en el privado, en procura de transitar hacia una senda de desarrollo resiliente al clima y bajo en carbono. A través de esta política, se busca que se aminoren los impactos asociados a la ocurrencia acelerada del cambio climático y que simultáneamente 
ofrezca luces sobre formas de aprovechamiento de las ventanas de oportunidad que este suceso genera. Lo anterior en relación con la pretensión que a largo plazo el país sea carbono neutral ${ }^{68}$.

Para tal efecto, ese macroobjetivo es desglosado en cuatro objetivos específicos los cuales precisan las acciones a adelantar para su materialización de la siguiente forma:

1. Orientar la gestión del cambio climático en segmentos de desarrollo preferentes de conformidad con su nivel de urgencia, priorizando aquellos donde confluyen instancias territoriales y sectoriales para la atención a asuntos relacionados con la afectación a población vulnerable y atinentes a adaptación y mitigación de GEI. Esto es: desarrollo urbano, desarrollo rural, desarrollo mineroenergético y desarrollo de infraestructura estratégica.

2. Conducir la gestión del cambio climático hacia el manejo sustentable y la preservación de los ecosistemas, así como de los servicios en estos originados, con el propósito de obtener metas comunes en los territorios en la reducción de la vulnerabilidad de la población y de sus actividades económicas.

3. Crear condiciones facilitadoras de la producción en ciencia, tecnología, información e innovación requeridas como insumo para transitar hacia la senda de desarrollo resiliente al clima y bajo en carbono.

4. Concebir las modificaciones institucionales a nivel político administrativo a favor del incremento en la efectividad de la gestión del cambio climático.

En ese contexto se trazan dos metas para ratificar los compromisos internacionales adquiridos. La primera es la de desarrollo bajo en carbono en dos modalidades, condicionada a su ejecución en sus máximos en el mediano y largo plazo por el respaldo internacional, de conformidad con lo previsto en el Acuerdo de París $(2015)^{69}$. Para lograrlo, el país se debe acoger a un aminoramiento progresivo en las emisiones de GEI tasado en mínimo de $20 \%$ y extremo de $30 \%$ respecto a las emisiones proyectadas para 2030. Esto es un el rango de 268 y 234 Mton de CO. en consideración a su registro de 224 Mton CO. en $2010^{70}$.

La segunda de las metas está delineada más no especificada en la política pública. Esta se refiere al desarrollo resiliente al clima, sobre lo cual preceptúa que su fijación competerá a la Comisión Intersectorial de Cambio Climático (CICC) con un monitoreo y posterior evaluación acerca de su efectividad en el tratamiento del impacto de los efectos del cambio climático, estableciendo, al mismo tiempo, que estos serán los referentes para medir el progreso de los entes territoriales y sectoriales en sus labores de adaptación a través del contraste entre las disposiciones relativas a este y su nivel de consecución en los respectivos planes de desarrollo establecidos para cada periodo de gobierno ${ }^{71}$.

En lo concerniente a los responsables, estos se sujetan a la regla de corresponsabilidad y complementariedad en sus labores como lo reseña la mencionada Ley 1931 de $2018^{72}$. No obstante, esto no es óbice para que, en aras de la claridad en la exigencia de deberes, se endilguen funciones a instituciones o instancias político administrativas, como resulta ser el caso de la CICC como delegada de la elaboración, aprobación y evaluación in extenso de la política; del IDEAM como encomendado de emisiones de GEI; y los Ministerios como delegados para la cofinanciación de la gestión del cambio climático en su respectivo sector ${ }^{73}$.

\section{Líneas de acción}

La Política Nacional de Cambio Climático se vale de la articulación de medidas procedimentales planificadas de índole territorial, sectorial e instrumental correspondientes a la previsión de objetivos que esta se propone. De esta manera busca garantizar la satisfacción de la interdependencia en pro de la efectiva incidencia en la toma de decisiones sectoriales, del desarrollo y de la ordenación sustentable del territorio con el fin de transitar hacia una senda de progreso resiliente al clima y bajo en carbono ${ }^{74}$. 
La primera de estas medidas es la "Estrategia de desarrollo rural resiliente al clima y bajo en carbono", la cual pretende saldar los atrasos en los procesos productivos de este segmento y promover su competitividad, reordenar la distribución de áreas geográficas para actividades económicas acorde a sus potencialidades, a saber, una agricultura más sustentable, una ganadería que no desate la degradación de los suelos, la pesca respetuosa tanto de los cuerpos hidrográficos como del criterio de seguridad alimentaria, y la promoción de iniciativas de reforestación; contribuyendo así a la reducción en las emisiones de GEI ${ }^{75}$.

La segunda es la "Estrategia de desarrollo urbano resiliente al clima y bajo en carbono", acorde con la predominante demografía urbana del país que representa el $76 \%$ de la población nacional, la concentración de gran parte de las emisiones de GEI en las urbes con relación al carácter masivo del transporte, el tratamiento de sus residuos y la amenaza latente de inundaciones por el irremediable asenso del nivel del $\operatorname{mar}^{76}$.

Así las cosas, la iniciativa aborda la vulnerabilidad al cambio climático tanto de las viviendas como del sector de la infraestructura en general y los ecosistemas naturales de las ciudades. Cuenta con herramientas como la provisión de saneamiento básico mediante sistemas de acueducto y alcantarillado, incentivos al uso ambientalmente consciente del agua, el fomento del reciclaje y la instauración transportes amigables con las condiciones de emisión de $\mathrm{GEI}^{77}$.

En tercer lugar, figura la "Estrategia de Desarrollo minero-energético bajo en carbono y resiliente al clima”, que atiende a que Colombia es el principal productor latinoamericano de Carbón y quinto exportador de dicho mineral en la región. Paradójicamente, las emisiones de GEI en este segmento presentaban una tendencia a la baja que se observa se invertirá, precisamente por una medida de reducción a la vulnerabilidad del sistema de generación de energía ante variaciones hidrológicas, por lo cual se fomentó la operatividad de centrales termoeléctricas ${ }^{78}$.

Ante esa dicotomía y la alta dependencia económica del país a los combustibles fósiles se apela al empleo de medidas como la expansión de la oferta de energía eléctrica nacional, el cambio al uso de biocombustibles con baja huella de carbono, el aprovechamiento de energías renovables y la gestión de las llamadas emisiones fugitivas de las actividades de minas e hidrocarburos, alusivas a aquellas que escapan del sistema de captación por mal diseño o desperfectos ${ }^{79}$.

En cuarto lugar, está la "Estrategia de Desarrollo de infraestructura estratégica baja en carbono y resiliente al clima" que atinente a las iniciativas de obras interregionales sobre transporte en cuanto a su generación de agravantes de los efectos del cambio climático. Es por esto que esta línea de acción busca incidir en la planificación, contratación y construcción de infraestructura para reducir la vulnerabilidad de las nuevas y antiguas edificaciones ${ }^{80}$.

Para tal propósito, dispone de medios como la promoción del esquema evitar-cambiar-mejorar, la incorporación de la variable de cambio climático en el diseño de la infraestructura de transporte y la evaluación de la infraestructura de transporte existente para la atenuación de su vulnerabilidad frente a los efectos de este fenómeno ${ }^{81}$.

Por último, se tiene la "Estrategia para el manejo y conservación de ecosistemas y sus servicios ecosistémicos para el desarrollo bajo en carbono y resiliente al clima” bajo la consigna del rol insustituible de la sanidad de los ecosistemas para la mitigación del cambio climático. Con esta iniciativa busca diagnosticar el estado de estos ecosistemas para tener suficientes elementos de juicio sobre las necesidades que estos ostentan y proceder de conformidad, apoyándose para tal efecto en los institutos científicos del SINA ${ }^{82}$.

\section{Conclusiones}

A nivel internacional y nacional se evidencia una evolución en respuesta al fenómeno de cambio climático, que va de una concentración de esfuerzos para la reducción de los gases de efecto invernadero (mitigación) 
con el fin de lograr combatir el cambio climático en los años noventa y principios del 2000, a una mezcla de mitigación y medidas de adaptación a los efectos del clima, como resultado de los impactos del mismo, a partir del año 2009.

En relación con los avances en política de cambio climático en Colombia, se evidencian tres períodos. Un primer período va desde el año de 1994 hasta 2010: la visión del país frente al cambio climático fue predominantemente económica; se enfocó en obtener provecho financiero de los proyectos relacionados con la reducción de gases de efectos invernadero.

Un segundo período correspondiente a los años 2010-2014, resultado del fenómeno de "La Niña" que azotó el país a finales del año 2010. Por los impactos y los desastres por los que pasó el país, la visión frente al fenómeno se enfocó en la adaptación y la gestión del riesgo de desastres.

El tercer período, correspondiente a los años 2014-2018, estuvo marcado por la necesidad y obligación como país de adherir y cumplir con los nuevos compromisos internacionales: el Convenio de Paris y los Objetivos de Desarrollo Sostenible, y lo relacionado con las ciudades, la nueva Agenda Urbana Global que aboga por el reconocimiento del derecho a la ciudad para la consecución de un hábitat sostenible. Se expide “Tercera comunicación nacional de cambio climático" (2015), la Política Nacional de Cambio Climático (2017) y la Ley 1931 de 2018. A partir del año 2018, se inicia la etapa de implementación de la Política Nacional y de la Ley de Cambio Climático.

Para lograr la efectividad de la Política Nacional de Cambio Climático y la Ley 1931 de 2018 es necesario:

a) La articulación efectiva de las entidades que integran el Estado colombiano.

b) Asignar rubros presupuestales en materia de cambio climático en las entidades públicas de todos los niveles, debido a la transversalidad de la Política Nacional de Cambio Climático.

c) Incluir la mitigación y adaptación en los instrumentos de planificación del territorio, desarrollo socioeconómico y la gestión del riesgo de desastres.

d) Socializar y sensibilizar a la comunidad sobre la Política Nacional de Cambio Climático, junto con la materialización efectiva en la construcción e implementación de los Planes Integrales de Gestión de. Cambio Climático Sectoriales y Territoriales. (PIGCCS. Y PIGCCT).

\section{Bibliografía}

Acuerdo de París [Naciones Unidas]. 12 de diciembre de 2015. https://unfccc.int/sites/default/files/spanish_paris_ agreement.pdf

Alejandra de Vengoechea, Las cumbres de las Naciones Unidas sobre Cambio Climático, Fundación Friedrich Ebert (FES) (2012). https://library.fes.de/pdf-files/bueros/la-energiayclima/09155.pdf

Arlene Tickner \& Sebastián Bitar, Nuevos enfoques para el estudio de las relaciones internacionales de Colombia (Ediciones Uniandes, 2017).

Banco de Desarrollo de América Latina, Índice de vulnerabilidad y adaptación al cambio climático en la región de América Latina y el Caribe (2014). http://scioteca.caf.com/handle/123456789/517

Comisión Económica para América Latina y el Caribe, América Latina y el Caribe: Estimaciones y proyecciones de población (2019). https://www.cepal.org/es/temas/proyecciones-demograficas/estimaciones-proyecciones-po blacion-total-urbana-rural-economicamente-activa

Gaceta No. 669 [Congreso de la República]. Exposición de Motivos Proyecto de Ley No. 73 de 2017 (Senado). Antecedentes Legislativos de la Ley 1931 de 2018 Por La Cual Se Establecen Directrices Para La Gestión Del Cambio Climático (2017).

Consejo Nacional de Política Económica y Social, Documento CONPES 3700. Estrategia institucional para la articulación de políticas y acciones en materia de cambio climático en Colombia (2011). https://colaboracion. dnp.gov.co/CDT/Conpes/Econ\%C3\%B3micos/3700.pdf 
Consejo Nacional de Política Económica y Social, Documento CONPES 3819. Política Nacional para la consolidación del sistema de ciudades en Colombia. (2014).

Convención Marco De Las Naciones Unidas Sobre El Cambio Climático (CMNUCC) (1992). https://unfccc.int/ resource/docs/convkp/convsp.pdf

Current World Population (Consultado el 30 de octubre de 2019). https://www.worldometers.info/es/

Decreto 298 de 2016. Por el cual se establece la organización y funcionamiento del Sistema Nacional de Cambio Climático y se dictan otras disposiciones. 24 de febrero de 2016. DO. No. 49796.

Dennis Meadows, Donella Meadows \& Jørgen Randers, Los límites del crecimiento: informe al club de roma sobre el predicamento de la humanidad (Fondo de Cultura Económica, 1972).

Dennis Meadows, Donella Meadows \& Jørgen Randers, Más allá de los límites del crecimiento (Aguilar, Taurus y Alfaguara, 1992).

Departamento Administrativo Nacional de Estadísticas (DANE), Resultados Censo Nacional de Población y Vivienda (2018). https://www.dane.gov.co/index.php/estadisticas-por-tema/demografia-y-poblacion/censo-nacional-d e-poblacion-y-vivenda-2018

Departamento Nacional de Planeación (DPN), Bases Plan de Desarrollo Prosperidad para Todos (2011). https://colaboracion.dnp.gov.co/CDT/PND/Bases\%20PND\%202010-2014\%20Versi\%C3\%B3n\%2 05\%2014-04-2011\%20completo.pdf

Departamento Nacional de Planeación (DPN), Plan Nacional de Adaptación al Cambio Climático. ABC: Adaptación Bases Conceptuales. Marco conceptual y lineamientos (2012). https://www.minambiente.gov.co/images/cambioclimatico/pdf/Plan_nacional_de_adaptacion/1.Pl an_Nacional_de_Adaptaci\%C3\%B3n_al_Cambio_Clim\%C3\%A1tico.pdf

Departamento Nacional de Planeación, Instituto de Hidrología, Meteorología y Estudios Ambientales, Ministerio de Ambiente y Desarrollo Sostenible, Sistema Nacional de Gestión del Riesgo de Desastres \& Unidad Nacional para la Gestión del Riesgo de Desastres, Hoja de ruta para la elaboración de los planes de adaptación dentro del Plan Nacional de adaptación al cambio climático (2013). http://comunidadpnacc.com/wp-content/uploads/2019/0 4/2._hoja_ruta_planes_adaptacion_v_0.pdf

Felipe Arbouin-Gómez, Reflexiones sobre la naturaleza del derecho urbanistico y propuesta de definición, Vniversitas, $\mathrm{n}$. ${ }^{\circ}$ 138, 1-16 (2019). https://doi.org/10.11144/Javeriana.vj138.rndu

Global Environmental Facility, Instituto de Hidrología, Meteorología y Estudios Ambientales, Ministerio de Vivienda y Desarrollo Territorial \& Programa de las Naciones Unidas para el Desarrollo, Segunda Comunicación nacional ante la convención marco de las naciones unidas sobre el cambio climático (2009). https://www.minambiente.g ov.co/index.php/comunicaciones-nacionales-de-cambio-climatico/segunda-comunicacion

Global Footprint Network, Global Footprint Network promotes real-world solutions that \#MoveTheDate, accelerating the transition to one-planet prosperity (29 de julio, 2019). https://www.footprintnetwork.org/2019/07/23/pre ss-release-july-2019/

Instituto de Hidrología, Meteorología y Estudios Ambientales, Ministerio de Ambiente y Desarrollo Sostenible, Cancillería de Colombia, Departamento Nacional de Planeación, Programa de Naciones Unidas Para el Desarrollo \& Global Environmental Facility, Tercera Comunicación Nacional de Colombia a la Convención de las Naciones Unidas sobre Cambio Climático (2017). http://documentacion.ideam.gov.co/openbiblio/bvirtua 1/023731/TCNCC_COLOMBIA_CMNUCC_2017_2.pdf

Instituto de Hidrología, Meteorología y Estudios Ambientales, Programa de Naciones Unidas Para el Desarrollo, Ministerio de Ambiente y Desarrollo Sostenible, Departamento Nacional de Planeación \& Cancillería Colombiana, Inventario nacional y departamental de gases efecto invernadero (2016). http://documentacion.i deam.gov.co/openbiblio/bvirtual/023634/INGEI.pdf

Intergovernmental Panel on Climate Change (IPCC) (30 de octubre de 2019). https://archive.ipcc.ch/home_langu ages_main_spanish.shtml

Intergovernmental Panel on Climate Change (IPCC), Comunicado de prensa del IPCC (30 de octubre de 2019). ht tps://www.ipcc.ch/site/assets/uploads/2019/05/2019Refinment-PR-es.pdf 
Ley 1151 de 2007. Por la cual se expide el Plan Nacional de Desarrollo 2006-2010. 25 de julio de 2007. DO. No. 46700.

Ley 1450 de 2011. Por la cual se expide el Plan Nacional de Desarrollo, 2010-2014. 16 de junio de 2011. DO. No. 48102.

Ley 1753 de 2015. Por la cual se expide el Plan Nacional de Desarrollo 2014-2018 “Todos por un nuevo país”. 9 de junio de 2015. DO. No. 49538.

Ley 1931 de 2018. Por la cual se establecen directrices para la gestión del cambio climático. 27 de julio de 2018. DO. No. 50667.

Ley 788 de 2002. Por la cual se expiden normas en materia tributaria y penal del orden nacional y territorial; y se dictan otras disposiciones. 27 de diciembre de 2002. DO. No. 45.046

Magda Ruiz, Norma Rubiano, Alejandro González, Thierry Lulle, Yolanda Bodnar, Sandra Velásquez, Sulma Cuervo \& Elizabeth Castellanos, Ciudad, espacio y población: El proceso de urbanización en Colombia (Universidad Externado de Colombia, 2007). https://www.uexternado.edu.co/wp-content/uploads/2017/04/Ciudad_espa cio_y_poblacion._El_proceso_de-Urbanizacion.pdf

María Camila Bustos, What Shapes Colombia's Foreign Position on Climate Change? Colombia Internacional, n. ${ }^{\circ} 94$, 27-51 (2018). https://doi.org/10.7440/colombiaint94.2018.02 (2017cee las Partes sobre su 24de desarrollo para la venidera regencia del Acuerdo de Parntran en la COP 22 o cumbre de

Mercedes Alcañiz, Cambios demográficos en la sociedad global, 14 Papeles de población, n. ${ }^{\circ}$ 57, 227-255 (2008). http: //www.scielo.org.mx/pdf/pp/v14n57/v14n57a11.pdf

Ministerio de Ambiente y Desarrollo Sostenible, Política nacional de cambio climático (2017). https://www.minambiente.gov.co/images/cambioclimatico/pdf/Politica_Nacional_de_Cambio_Cli matico___PNCC_/PNCC_Politicas_Publicas_LIBRO_Final_Web_01.pdf

Ministerio de Medio Ambiente, Instituto de Hidrología, Meteorología y Estudios Ambientales \& Programa de las Naciones Unidas para el Desarrollo, Primera Comunicación Nacional ante la Convención Marco de las Naciones Unidas sobre el Cambio Climático (2001). http://www.ideam.gov.co/documents/40860/219937/primera-co municacion--nacional/b99663bb-9023-47d1-b54a-41f74cca0b1e

Ministerio de Medio Ambiente \& Departamento Nacional de Planeación, Resumen Ejecutivo Lineamientos de Política de Cambio Climático (2002). https://www.preventionweb.net/files/21403_15719lineamientospoliticanaciona lca.pdf

Organización de las Naciones Unidas, Convención Marco sobre el Cambio Climático, Informe de la Conferencia de las Partes sobre su tercer período de sesiones (Kioto, 1997). https://unfccc.int/resource/docs/spanish/cop3/g 9860818.pdf

Organización de las Naciones Unidas, Convención Marco sobre el Cambio Climático, Informe de la Conferencia de las Partes sobre su $21^{\circ}$ período de sesiones (París, 2015). https://unfccc.int/resource/docs/2015/cop21/spa/l0 9s.pdf

Organización de las Naciones Unidas, Convención Marco sobre el Cambio Climático, Informe de la Conferencia de las Partes sobre su $22^{\circ}$ periodo de sesiones (Marrakech, 2016).

Organización de las Naciones Unidas, Convención Marco sobre el Cambio Climático, Informe de la Conferencia de las Partes sobre su $23^{\circ}$ período de sesiones (Bonn, 2017). https://unfccc.int/sites/default/files/resource/docs/ 2017/cop23/spa/11a01s.pdf

Organización de las Naciones Unidas, Convención Marco sobre el Cambio Climático, Informe de la Conferencia de las Partes sobre su $24^{\circ}$ periodo de sesiones (Katowice, 2018). https://unfccc.int/sites/default/files/resource/10s.pdf

Paul R. Earlich, The Population Bomb (1.a ed., Ballantine Books, 1968).

Organización de las Naciones Unidas, Protocolo de Kioto de la convención marco de Las Naciones Unidas sobre el cambio climático (1997). https://unfccc.int/resource/docs/convkp/kpspan.pdf

United Nations, Framework Convention on Climate Change (30 de octubre, 2019). https://www.un.org/en/sections/ issues-depth/climate-change/index.html 
United Nations, World Population Prospects: Highlights (17 de junio, 2019). https://www.un.org/development/desa/ publications/world-population-prospects-2019-highlights.html\#: :text=The\%20world's\%20population\%20i s\%20expected,United\%20Nations\%20report\%20launched\%20today.

Warren S. Thompson, Population, 34 American Sociological Review, n. ${ }^{\circ}$ 6, $959-975$ (1929). https://www.jstor.org/s table/2765883

WCRP History (consultado el 30 de octubre, 2019). https://www.wcrp-climate.org/about-wcrp/about-history

WCRP Mission (consultado el 30 de octubre, 2019). https://www.wcrp-climate.org/about-wcrp/wcrp-overview

\section{Notas}

* Artículo de investigación. Resultado del avance de la investigación doctoral denominada: "Sostenibilidad ambiental urbana en el Caribe Colombiano: análisis jurídico-administrativo de las actuaciones realizadas por los Distritos del Caribe colombiano para lograr la mitigación y adaptación al cambio climático".

1 Current World Population (Consultado el 30 de octubre de 2019).

2 Mercedes Alcañiz, Cambios demográficos en la sociedad global, 14 Papeles de población, n. ${ }^{\circ}$ 57, 227-255 (2008).

3 Warren $S$. Thompson desarrolló esta teoría para analizar los cambios que habían experimentado en los últimos doscientos años las sociedades industrializadas de su tiempo con respecto a las tasas de natalidad y de mortalidad. Warren S. Thompson, Population, 34 American Sociological Review, n. 6, 959-975 (1929).

4 Global Footprint Network, Global Footprint Network promotes real-world solutions that \#MoveTheDate, accelerating the transition to one-planet prosperity (29 de julio, 2019). Esta ONG ha revelado en su informe anual que el presupuesto ecológico se gasta cada vez de manera más prematura, ritmo que demanda la tenencia de 1,75 planetas a disposición de la humanidad.

Tal como fue advertido en Dennis Meadows, Donella Meadows \& Jørgen Randers, Los límites del crecimiento: informe al club de roma sobre el predicamento de la humanidad (Fondo de Cultura Económica, 1972) y corroborado en versiones ratificatorias posteriores como Dennis Meadows, Donella Meadows \& Jørgen Randers, Más allá de los límites del crecimiento (Aguilar, Taurus y Alfaguara, 1992).

Desde el informe Meadows (1972) se ha señalado que, en algún momento en el siglo XXI, el planeta llegará a un punto de colapso de la capacidad del mismo para absorber la actividad antrópica. Lo anterior coincide con los hallazgos de Wackernhagel y Rees (1996), quienes han establecido la biocapacidad o diseño ecológico del planeta en 3.500 millones de personas; hoy la población mundial está en el orden de los 7.740. millones y se esperan 9.000 millones a 2050. Current World Population (Consultado el 30 de octubre de 2019).

Paul R. Earlich, The Population Bomb (1. a ed., Ballantine Books, 1968).

7 En Colombia una reseña al respecto se halla en Magda Ruiz, Norma Rubiano, Alejandro González, Thierry Lulle,

8 Yolanda Bodnar, Sandra Velásquez, Sulma Cuervo \& Elizabeth Castellanos, Ciudad, espacio y población: El proceso de urbanización en Colombia, 71 (Universidad Externado de Colombia, 2007).

9 United Nations, World Population Prospects: Highlights (17 de junio, 2019)

10 Departamento Administrativo Nacional de Estadísticas (DANE), Resultados Censo Nacional de Población y Vivienda, 66 (2018).

11 Íd.

12 Consejo Nacional de Política Económica y Social, Documento CONPES 3819. Política Nacional para la consolidación del sistema de ciudades en Colombia, 69 (2014).

13 Comisión Económica para América Latina y el Caribe, América Latina y el Caribe: Estimaciones y proyecciones de población, 7 (2019).

14 Consejo Nacional de Política Económica y Social, Documento CONPES 3819, op. cit., 69.

15 Conforme a esto, la disciplina urbanística adopta como esencial a su concepto el tema ambiental. Véase: Felipe ArbouinGómez, Reflexiones sobre la naturaleza del derecho urbanistico y propuesta de definición, Vniversitas, n. ${ }^{\circ}$ 138, 1-16 (2019).

16 Instituto de Hidrología, Meteorología y Estudios Ambientales, Programa de Naciones Unidas Para el Desarrollo, Ministerio de Ambiente y Desarrollo Sostenible, Departamento Nacional de Planeación \& Cancillería Colombiana, Inventario nacional y departamental de gases efecto invernadero, 73 (2016).

17 De acuerdo con la III Comunicación de Colombia a la Convención Marco de Cambio Climático para dar reporte del cumplimiento de los compromisos adquiridos por el país, Colombia aumentó la producción de GEI, al pasar del 0,34 a 0,41\% del total de los GEI, lo cual equivale a 22 gigatoneladas de dióxido de carbono CO2. A pesar de que sigue siendo una aportación insipiente, preocupa el hecho de que la concentración de GEI en vez de disminuir, aumenta. 
18 Banco de Desarrollo de América Latina, Índice de vulnerabilidad y adaptación al cambio climático en la región de América Latina y el Caribe, 212 (2014).

19 Alejandra de Vengoechea, Las cumbres de las Naciones Unidas sobre Cambio Climático, Fundación Friedrich Ebert (FES) (2012).

20 WCRP History (consultado el 30 de octubre, 2019). https://www.wcrp-climate.org/about-wcrp/about-history

21 WCRP Mission (consultado el 30 de octubre, 2019). https://www.wcrp-climate.org/about-wcrp/wcrp-overview

22 Intergovernmental Panel on Climate Change (IPCC) (30 de octubre de 2019). https://archive.ipcc.ch/home_langua ges_main_spanish.shtml

23 Intergovernmental Panel on Climate Change (IPCC), Comunicado de prensa del IPCC (30 de octubre de 2019). http s://www.ipcc.ch/site/assets/uploads/2019/05/2019Refinment-PR-es.pdf

24 Esto, con un clima adverso y contra fáctico debido a agitadores del negacionismo y la reciente salida de EE. UU. del mismo, comandada por Donald Trump.

25 United Nations, Framework Convention on Climate Change (30 de Octubre, 2019). https://www.un.org/en/sections/ issues-depth/climate-change/index.html

26 Convención Marco De Las Naciones Unidas Sobre El Cambio Climático (CMNUCC), Art. 7 (1992).

27 Organización de las Naciones Unidas, Convención Marco sobre el Cambio Climático, Informe de la Conferencia de las Partes sobre su tercer período de sesiones, 53 (Kioto, 1997).

28 El mecanismo de desarrollo limpio es el único instrumento abierto para la cooperación entre estados desarrollados con metas de reducción de emisiones y los países en desarrollo sin metas de reducción. En Colombia esta figura ha sido utilizada para promover todo tipo de proyectos.

29 Organización de las Naciones Unidas, Convención Marco sobre el Cambio Climático, Informe de la Conferencia de las Partes sobre su $21^{\circ}$ período de sesiones, 40 (París, 2015).

30 Acuerdo de París [Naciones Unidas]. 12 de diciembre de 2015. Art. 2.

31 Organización de las Naciones Unidas, Convención Marco sobre el Cambio Climático, Informe de la Conferencia de las Partes sobre su $22^{\circ}$ período de sesiones, 35 (Marrakech, 2016).

32 Organización de las Naciones Unidas, Convención Marco sobre el Cambio Climático, Informe de la Conferencia de las Partes sobre su $23^{\circ}$ período de sesiones, 31 (Bonn, 2017).

33 Organización de las Naciones Unidas, Convención Marco sobre el Cambio Climático, Informe de la Conferencia de las Partes sobre su $24^{\circ}$ período de sesiones, 29 (Katowice, 2018).

34 De acuerdo con cálculos del Departamento Nacional de Planeación, la atención al fenómeno de la niña ocurrido entre finales de 2010 y mediados de 2011 le costó al país cerca de 26 billones de pesos. Departamento Nacional de Planeación (DPN), Bases Plan de Desarrollo Prosperidad para Todos, 861 (2011).

35 Ley 164 de 1994 "Por medio de la cual se aprueba la "Convención Marco de las Naciones Unidas sobre el Cambio Climático"

36 Instrumento internacional que, para el caso de Colombia, significó la búsqueda de beneficios de cooperación internacional en materia ambiental y darse una posición en el espectro geopolítico mundial, para lo cual se valió de su fuerte activismo y conocimiento tanto técnico como político en la materia. Ver María Camila Bustos, What Shapes Colombia's Foreign Position on Climate Change? Colombia Internacional, n. ${ }^{\circ}$ 94, 27-51 (2018); Arlene Tickner \& Sebastián Bitar, Nuevos enfoques para el estudio de las relaciones internacionales de Colombia, 363 (Ediciones Uniandes, 2017).

37 Por medio de la Ley 629 de 2000.

38 El MDL fue definido en el artículo 12 del Protocolo de Kioto y, de conformidad con sus numerales 2 y 3 , este mecanismo permite a las partes no incluidas en el anexo I[38] beneficiarse de las actividades de proyectos que tengan como resultado reducciones certificadas de emisiones y, en el mismo sentido, las partes incluidas en el anexo I, utilizar las reducciones certificadas de emisiones resultantes de esas actividades de proyectos para contribuir al cumplimiento de una parte de sus compromisos cuantificados de limitación y reducción de las emisiones contraídas. Organización de las Naciones Unidas, Protocolo de Kioto de la convención marco de Las Naciones Unidas sobre el cambio climático (1997).

39 Ministerio de Medio Ambiente, Instituto de Hidrología, Meteorología y Estudios Ambientales \& Programa de las Naciones Unidas para el Desarrollo, Primera Comunicación Nacional ante la Convención Marco de las Naciones Unidas sobre el Cambio Climático, 267 (2001).

40 Ministerio de Medio Ambiente \& Departamento Nacional de Planeación, Resumen Ejecutivo Lineamientos de Política de Cambio Climático (2002).

41 (Art. 17 Comercio de derechos de emisión)

42 En esta normativa se establece como renta exenta la causada en la tramitación, obtención y venta de certificados de emisión de bióxido de carbono en el marco de proyectos de generación de energía eólica, a partir de biomasa y residuos agrícolas (Art. 18 Modificatorio del 207-2 del Estatuto Tributario). 
43 Instituido en la sesión No. XX del 29 de agosto de 2003 del Consejo Nacional Ambiental, previa recomendación por parte del Consejo Nacional de Política Económica y Social en el Documento CONPES 3242 de 2003.

44 Ley 1151 de 2007. Por la cual se expide el Plan Nacional de Desarrollo 2006-2010. 25 de julio de 2007. DO. No. 46700. Art. 6, Numeral 5.

45 Global Environmental Facility, Instituto de Hidrología, Meteorología y Estudios Ambientales, Ministerio de Vivienda y Desarrollo Territorial \& Programa de las Naciones Unidas para el Desarrollo, Segunda Comunicación nacional ante la convención marco de las naciones unidas sobre el cambio climático, 407 (2009).

46 Íd.

47 Ley 1450 de 2011. Por la cual se expide el Plan Nacional de Desarrollo, 2010-2014. 16 de junio de 2011. DO. No. 48102. Art. 3.

48 Con este se da un viraje en el entendimiento ya no rentístico del asunto, sino en términos de adaptación y mitigación frente a lo cual es imperioso advertir. No resulta de la conciencia al respecto, sino de lo calamitoso que se tornó el fenómeno de la niña que azotó al país entre esos tiempos.

49 Ley 1450 de 2011. Art. 16.

50 Ley 1450 de 2011. Art. 217.

51 Consejo Nacional de Política Económica y Social, Documento CONPES 3700. Estrategia institucional para la articulación de políticas y acciones en materia de cambio climático en Colombia, 75 (2011).

52 Departamento Nacional de Planeación (DPN), Plan Nacional de Adaptación al Cambio Climático. ABC: Adaptación Bases Conceptuales. Marco conceptual y lineamientos, 80 (2012).

53 La adopción de este instrumento fue derogada por el Artículo 267 de la Ley 1753 de 2015, Plan Nacional Desarrollo (2014-2018) del Gobierno de Juan M. Santos.

54 Departamento Nacional de Planeación, Instituto de Hidrología, Meteorología y Estudios Ambientales, Ministerio de Ambiente y Desarrollo Sostenible, Sistema Nacional de Gestión del Riesgo de Desastres \& Unidad Nacional para la Gestión del Riesgo de Desastres, Hoja de ruta para la elaboración de los planes de adaptación dentro del Plan Nacional de adaptación al cambio climático, 103 (2013).

55 Ley 1753 de 2015. Por la cual se expide el Plan Nacional de Desarrollo 2014-2018 “Todos por un nuevo pás". 9 de junio de 2015. DO. No. 49538. Art. 5.

56 Ley 1753 de 2015. Por la cual se expide el Plan Nacional de Desarrollo 2014-2018 “Todos por un nuevo país". 9 de junio de 2015. DO. No. 49538. Art. 155.

57 Ley 1753 de 2015. Por la cual se expide el Plan Nacional de Desarrollo 2014-2018 “Todos por un nuevo país”. 9 de junio de 2015. DO. No. 49538. Art. 170.

58 Planeación, Programa de Naciones Unidas Para el Desarrollo \& Global Environmental Facility, Tercera Comunicación Nacional de Colombia a la Convención de las Naciones Unidas sobre Cambio Climático, 546 (2017).

59 Decreto 298 de 2016. Por el cual se establece la organización y funcionamiento del Sistema Nacional de Cambio Climático y se dictan otras disposiciones. 24 de febrero de 2016. DO. No. 49796. Art. 2.

60 Decreto 298 de 2016. Art. 1.

61 Consejo Nacional de Política Económica y Social, Documento CONPES 3700, op. cit., 75.

62 Íd.

63 Decreto 298 de 2016. Art 4.

64 Ministerio de Ambiente y Desarrollo Sostenible, Política nacional de cambio climático, 148 (2017).

65 En esta legislación se amplía el concepto de cambio climático incorporado por la Ley 1523 de 2012 (Art. 4 No. 6) al insertar formas de medir su ocurrencia y ejemplificando sus manifestaciones (Art. 3 No. 4 L. 1931/18).

66 Gaceta No. 669 [Congreso de la República]. Exposición de Motivos Proyecto de Ley No. 73 de 2017 (Senado). Antecedentes Legislativos de la Ley 1931 de 2018 Por La Cual Se Establecen Directrices Para La Gestión Del Cambio Climático, 27 (2017).

67 Ley 1931 de 2018. Por la cual se establecen directrices para la gestión del cambio climático. 27 de julio de 2018. D.O. No. 50667. Art. 3.

68 Ministerio de Ambiente y Desarrollo Sostenible, op. cit., 148.

69 Aprobado mediante la Ley 1844 de 2017.

70 Ministerio de Ambiente y Desarrollo Sostenible, op. cit., 148.

71 Íd.

72 Ley 1931 de 2018. Por la cual se establecen directrices para la gestión del cambio climático. 27 de julio de 2018. D.O. No. 50667. Art. 2.

73 Ministerio de Ambiente y Desarrollo Sostenible, op. cit., 148.

74 Íd.

75 Íd.

76 Íd. 
77 Íd.

78 Íd.

79 Íd.

80 Íd.

81 Íd.

82 Íd.

Licencia Creative Commons CC BY 4.0

Para citar este articulo/To cite this article: Luis Magin Guardela Contreras, Evolución de la política de cambio climático en Colombia, 69 Vniversitas (2020). https://doi.org//10.11144/Javeriana.vj69.epcc 